\title{
ADAPTACIÓN Y TRANSMEDIALIDAD: CRÍTICA DE UNA OPOSICIÓN AGOTADA
}

\author{
ADAPTATION \& TRANSMEDIALITY: \\ A CRITIQUE OF AN EXHAUSTED OPOSITION
}

Nieves Rosendo SÁNCHeZ

Universidad de Granada

n.rosende@gmail.com

DOMingo SÁnCHEZ-MeSA MARTíneZ

Universidad de Granada

dsanchez@ugr.es

\begin{abstract}
RESUMEN: En este artículo se revisa el debate teórico sobre la relación y función de la adaptación en el tipo de diseño narrativo distribuido denominado transmedia storytelling (Jenkins 2003, 2006, 2009). Dicha revisión permite matizar y hasta corregir una oposición establecida en el primer momento de desarrollo del discurso mainstream sobre el TS (Jenkins 2003, Long 2007, Gomez 2007) según la cual la adaptación no sería un tipo de estrategia propia o recomendable en dicho tipo de narrativas. Siguiendo a Christy Dena, se identifican varias razones, de orden más bien pragmático, para aquella oposición inicial. Posteriormente se cuestiona la reducción del concepto de adaptación al de un grado inferior extremo en el espectro entre "repetición" y "expansión", para entenderla como práctica de transferencia cultural más amplia y compleja. El texto profundiza también en las consecuencias de este debate a la luz de la discusión previa del Proyecto de investigación Nar-Trans sobre la dimensión intermedial de la transmedialidad, la concepción desmaterializada de "medio" de la teoría sobre el transmedia storytelling y de la transmedialidad misma como tendencia centrada en la construcción de mundos narrativos a través del flujo aparentemente sin resistencia de contenidos demediados (Sánchez-Mesa y Baetens 2017; Baetens y Sánchez-Mesa 2019).
\end{abstract}

PALABRAS CLAVE: Transmedialidad; adaptación; transmedia storytelling; intermedialidad; demediación; mundos narrativos; expansión 
ABSTRACT: This article reviews critically the theoretical debate on the role of adaptation in transmedia storytelling as a kind of distributed narrative design across different media (Jenkins 2003, 2006, 2009). This revision finally contradicts and corrects an oposition established at an early stage of the mainstream discourse on transmedia storytelling (Jenkins 2003, Long 2007, Gomez 2007) according to which adaptation was not part or an adequate strategy for this kind of narratives. Following Christy Dena, various reasons are identified for such a dicotomy. Next, the limitation of adaptation as an extreme minimum degree between "repetition" and "expansion" is criticised in order to understand it as a broader and more complex cultural transfer practice. The text goes deeper into the consequences of this debate continuing the theoretical positions developed during the research project Nar-Trans, that is, tackling issues such as the intermedial dimension of transmedialization processes, the inmaterialization of media operated within the transmedia storytelling theory as transmediality itself turns out to be more depending on storyworlds building through the permanent flux of demediated contents (Sánchez-Mesa \& Baetens 2017; Baetens \& Sánchez-Mesa 2019).

KeyWords: Transmediality; Adaptation; Transmedia storytelling; InTERmeDiality; Demediation; StoryWORLDS, Expansion

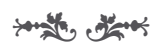

\section{Pré́mbulo}

A pesar de los síntomas de cierto agotamiento en el debate sobre el transmedia storytelling o la narrativa transmedial, ${ }^{1}$ la confusión e imprecisión con que se sigue haciendo uso de este término sigue invitándonos a profundizar y persistir en la aclaración del funcionamiento de este tipo de diseño narrativo (Pratten 2015: 3) y su categorización teórica. Incluso en términos lingüísticos y de traducción desde el término inglés (transmedia storytelling) venimos apostando por el uso del sintagma "narrativas transmediales" por las implicaciones que tiene sobre la noción de "medio" y de "adaptación" misma, por contraposición a los planteamientos dominantes en la teoría y la práctica mainstream del transmedia storytelling. En dicho escenario, el juego entre los límites y la hibridación de los rasgos específicos de cada medio -la misma Marsha Kinder, pionera en la identificación de la intertextualidad transmedial, ha llamado la atención sobre la necesidad de regresar a esta cuestión (Kinder 2014)- plantean nuevos retos a la Teoría literaria, a los Estudios comparados de medios (incluida la Litera-

\footnotetext{
1 Si bien podemos considerarlos sinónimos, no creemos que sean términos equivalentes (Sánchez-Mesa y Baetens 2015, 2017a).
} 
tura comparada), a la Teoría de la comunicación y a los Estudios culturales. La relevancia de nuestra llamada a una correcta teorización de la adaptación en relación a las narrativas transmediales viene corroborada, recientemente, por el reconocimiento por parte del mismo Henry Jenkins de la necesidad de establecer puentes entre las investigaciones en ambos dominios y que esas conexiones -añadiríamos nosotros- fueran suficientemente sólidas y rigurosas: ${ }^{2}$

Those of us who study transmedia (and fan fiction) and those who study adaptation are asking a related set of questions, though as of now we are often talking past each other, because our terminological and methodological assumptions lead us to underestimate the materials the other is studying. (Jenkins 2017)

\section{DialogizACIÓN DE UN DEBATE: TRANSMEDIA STORYTELLING VS. ADAPTACIÓN}

La adaptación, como estrategia de transferencia de contenido y de fidelización de la audiencia en torno a un mundo ficcional, ha despertado cierta controversia en el discurso sobre el transmedia storytelling (a partir de aquí, TS) desde un primer momento. Ya desde el propio artículo seminal de Henry Jenkins de 2003, este indicaba que, en el contexto industrial de grandes conglomerados de medios era necesario "a new model for co-creation-rather than adaptationof content that crosses media". Como veremos más adelante, esta postura del teórico de medios cambió, sin embargo, en los siguientes años. En relación a la exclusión de la adaptación del TS, los ejemplos más relevantes los encontramos aproximadamente en la primera década del siglo (Jenkins 2006, Long 2007, Evans 2011, Bourdaa 2013), mientras que en la frontera con la segunda década existe cierto acercamiento a una perspectiva más integradora que fue iniciado por Christy Dena (Dena 2009, 2018; Jenkins 2009, 2011, 2017; Scolari 2009, 2012, 2013, 2016; Parody 2011; Hutcheon y O'Flynn 2013; Aarseth 2016; Ryan 2017). Los argumentos más utilizados en contra de la adaptación son los de la redundancia y la repetición del relato, a favor del término expansión, diferenciando así el TS de la establecida práctica de la adaptación. También es destacable cómo, desde la propia industria, se ha buscado separar la adaptación del TS, con el ejemplo paradigmático de la definición de productor transmedia que hace el Producers Guild of America (2010), donde definen los elementos de una producción transmedia excluyendo precisamente la reutilización del contenido de un medio a otro.

En este debate, siempre hemos sostenido que la adaptación funciona de hecho como una de las piedras angulares de cualquier tipo de proceso de transmedialización (Baetens y Sánchez-Mesa 2015). Espen Aarseth coincide con este argumento colocando la adaptación "en el corazón" de toda franquicia crossgenre, al tiempo que entiende que tal vez el mismo término haya quedado algo

2 Este ha sido uno de los objetivos fundamentales del proyecto Nar-Trans desde sus inicios en 2014 y el objeto de uno de los paneles de discusión de cada una de las reuniones internacionales organizadas. 
superado siendo desplazado por el crossmedia storytelling (relato crosmedial) (Sánchez-Mesa et alii 2016: 13). Por otro lado, en la segunda edicion de $A$ Theory of Adaptation (2013), Linda Hutcheon y S. O'Flynn abren el radio de aplicación para dar cabida no solo a los llamados "heterocosmos" o mundos narrativos propios de los videojuegos, sino también a los relatos propios de las franquicias y también a las recreaciones que los fans hacen de los mundos transmediales, considerándolos como "a strategy of participation". En el capítulo final sobre adaptación y nuevos medios que escribe O'Flynn se subrayan los solapamientos que se producen entre adaptaciones y TS en casos paradigmáticos de adaptaciones como las series de televisión Sherlock (BBC), Game of Thrones (HBO), o la de films The Hunger Games (Lionsgate), a su vez objeto de adaptaciones generadas por los fans, como en el caso también del universo generado en Pottermore, a partir de la franquicia originada en las novelas de J.K. Rowling, lo que viene a complicar aún más el fenómeno (Hutcheon y O’Flynn 2013: 187).

Por su parte, Carlos A. Scolari se ha ocupado de la tensión TS vs. adaptación en varias ocasiones, la primera a propósito de la expansión transmedial del universo ficcional de Las tres mellizas (Scolari 2012), que tiene su origen en la literatura infantil de Roser Capdevila y Enric Larreula. ${ }^{3}$ Scolari se refiere al "conflicto" entre expansión transmedial y adaptación (2012: 50) y, haciendo gala de la base semiótica de su artículo, habla de "traducción intersemiótica" (siguiendo a Jakobson 1985), subrayando la centralidad de la actividad traductora en el sistema cultural, en la línea del teórico de la Escuela de Tartu, Peter Torop.

En su breve repaso de los hitos más influyentes en este debate (Jenkins 2006, 2009, 2011; Long 2007; Dena 2009) Scolari destaca y opta por la propuesta de Dena y su invitación a que el TS sea lo suficientemente flexible como para incorporar adaptaciones que de algún modo enriquezcan y expandan un mundo narrativo, y apoyándose en Jenkins (2011) sitúa la adaptación y la expansión como dos polos entre los que se produce esta práctica. Si bien podemos estar de acuerdo básicamente con esta aserción, la diferencia con nuestra postura descansa en la estrechez del concepto de adaptación, que aquí se identifica precisamente con uno de los polos de ese espectro: el que se supone más cercano del texto original, es decir con la idea de "repetición". La adaptación no es, en nuestra concepción, una práctica estrecha o reducida de la "traducción intersemiótica" sino un conjunto de prácticas muy amplio, que puede y debe abarcar aquellas definidas o descritas con otros términos como "versión", "expansión", "compresión", "secuela", "precuela", es decir, todo el dominio abarcado por la transposición diegética (Genette, 1982) o la transducción (Dolezel, 1986).

En "Transmedia (Storytelling?): A polyphonic critical review" (Sánchez-Mesa et al. 2016), Scolari claramente aboga por la inclusión de la adaptación dentro del TS, tanto desde un punto de vista analítico como de producción:

\footnotetext{
${ }^{3}$ Libros, serie de dibujos animados, web y cd interactivos, adaptación teatral, aplicación iPad; todo ello a propósito de la aparición en ese universo de la figura de Antonio Gaudí.
} 


\begin{abstract}
... we can't analyse Harry Potter's narrative world and exclude the movies just because they are adaptations of the books! Anarrative world includes adaptations, expansions and... compressions! Also trailers, sneak-peeks and recapitulations (often made by fans) are part of the TN world and, accordingly we can't rule them out of our research. (Sánchez-Mesa et al. 2016: 13)
\end{abstract}

Si bien Scolari es taxativo al respecto de la cuestión que nos ocupa, la distinción misma que establece entre adaptación, expansión y compresión denota un concepto de adaptación que no coincide con el que nosotros proponemos, el cual abarcaría transferencias de un texto anterior que supusieran una "expansión" o un "compresión", imposibles sin las operaciones propias de una adaptación.

En este mismo artículo, Robert Pratten, desde la perspectiva del productor transmedia, en sintonía con otros productores de relevancia como Jeff Gomez (2007) y también desde un concepto reducido de la adaptación, considera que esta "may act as a short-cut to slide back into experiences" y en este sentido sostiene que "I find other courses of action more rewarding than basically copying a story that's already been told" (Sánchez-Mesa et al. 2016: 13). Esta falta de atención sobre la adaptación ya era evidente en Getting Started in Transmedia Storytelling, del mismo Pratten (2011, 2015).

Christy Dena, en una reciente publicación (2018) titulada "Transmedia Adaptation. Revisiting the Non-Adaptation Rule", pone en perspectiva esta tendencia, tanto académica como profesional, en el que la adaptación parece no tener cabida, recogiendo distintas aportaciones que aquí ya hemos apuntado en contra de la inclusión de la adaptación. Para esta autora, investigadora y creativa en este dominio narrativo, el motivo por el que esta exclusión siga dándose tanto en la literatura académica como en la propia creación de las narrativas transmediales es que dicha exclusión cumple con tres funciones claras: a) establecer una distinción entre TS y las prácticas multiplataforma anteriores presentes en la industria, como diversas prácticas de márketing y licensing, productos como los spin-offs o las novelizaciones; b) proveer a los estudios de medios con una base narratológica introduciendo una nueva teoría, para diferenciar de una forma específica este área emergente de los muy establecidos estudios culturales; y c) una función pedagógica, de modo que sea sencillo explicar el fenómeno a los no iniciados en él mediante esta simple oposición o exclusión (Dena 2018: 198).

Esta última función, según la cual el TS se apoya antes en el concepto de expansión que en el proceso de adaptación como una forma pedagógica de introducir aquel primer concepto, ha tenido consecuencias como, por ejemplo, el establecimiento de tres plataformas como el número mínimo para desarrollar una narrativa transmedial (v.g. Producer's Guild of America). Sobre esta cuestión, Dena recoge las declaraciones de Jenkins (2011) en las que se lamenta de que los escritores de narrativas transmediales parecen preocuparse más por el número de plataformas que sobre la forma en que se relacionan estas, y cómo lo mismo sucede con los estudios académicos. En cuanto a la función de delimitación del área de estudios, de nuevo la diferenciación es el motivo por el cual se excluye la adaptación en el TS, y también una dinámica habitual de falsa sustitución de 
lo viejo por lo nuevo "transmedia is different to franchises, it is better because it extends stories" (Dena 2018: 199). Sabemos que una de las consecuencias de definir un objeto de estudio a través de determinados rasgos y características es la limitación en sí de la observación en perspectiva del mismo. El considerar que la adaptación no es característica del TS impide que tanto los creadores, como los investigadores y estudiantes presten atención al estudio de la misma en estos nuevos ámbitos. Esta exclusión inicial implica además una simplificación del fenómeno de la adaptación en virtud de los argumentos de redundancia y repetición del relato que no se sostienen ni teóricamente ni en vista del éxito de las adaptaciones en la industria del entretenimiento. Dena concluye advirtiendo sobre los efectos que la exclusión de la adaptación tiene para el estudio, la creación y la enseñanza del TS. Citando a Wells-Lassagne (2017:92) los estudios sobre adaptaciones pueden ayudar en el futuro a aquellos sobre TS respecto a cuestiones como, por ejemplo, la fidelidad, la "bestia negra" de los estudios de la adaptación. Por otro lado, los conceptos de familiaridad y novedad utilizados en el estudio de las narrativas televisivas, pueden proponer nuevos enfoques en el estudio y creación narrativas transmediales. Excluir la adaptación del TS, en su estudio, docencia y práctica, lleva a una jerarquización cuyos resultados indeseados son no comprender por completo el fenómeno y olvidar una serie de prácticas comunes en el diseño creativo (2018:203). El foco en las expansiones es necesario, por otro lado, pero el olvido de otras prácticas asociadas al TS no nos permite observar este fenómeno en perspectiva y en toda su potencial complejidad.

El propio Henry Jenkins, en su artículo "Adaptation, Extension, Transmedia" (2017), reflexiona sobre los efectos de la mencionada exclusión, pero también sobre la oposición entre expansión y adaptación. Reconsiderando su posición original una década atrás sobre la adaptación, Jenkins admite que un estudio serio de la adaptación lleva inmediatamente a la conclusión de que esta hace una mayor o menor contribución única a la historia, seleccionando e interpretando el material, y utilizando de manera consciente las posibilidades del medio de destino. Sin embargo, para Jenkins el término expansión implica ir más allá del original: expandiendo por ejemplo el tiempo de la historia o desarrollando la historia de un personaje secundario. En la práctica, dice Jenkins, se trata de una cuestión de grado, como ha venido elaborando desde 2011: "In practice, the difference may be only a matter of degree -adding and subtracting scenes and characters in the case of a faithful adaptation, adding entire stories or corners of the universe in the case of a transmedia extensión" (Jenkins 2017).

Llamamos la atención sobre los mismos términos empleados en esta cita por Jenkins: "faithful adaptation". Es evidente que para él un término del sintagma acaba exigiendo o demandando al otro. En cualquier caso, Jenkins coincide con Christy Dena: el foco en la expansión fue una forma en que los teóricos del transmedia criticaron (siguiendo sus primeras definiciones, como hace por ejemplo su discípulo Geoffrey Long) la redundancia que destruía la calidad de muchas novelizaciones de franquicias de medios y que dejaba de lado otras posibilida- 
des creativas. El resultado de dicha operación ha sido apartar los estudios sobre la adaptación de los estudios sobre transmedia durante una década.

Para Jenkins, el concepto de creación de mundos (world building) ofrece la oportunidad de reconsiderar estas relaciones. En la creación de mundos se dan tanto operaciones de adaptación como de expansión, con contribuciones originales. Las expansiones, además, se legitiman en la forma en que encajan con el mundo previamente establecido y nos devuelven a él con una nueva visión del mismo. Los teóricos transmedia, según el autor, distinguen entre elementos u obras que contribuyen a la continuidad y otros que lo que contribuyen es a la multiplicidad de mundos, a una reinvención radical del existente. Y precisamente las obras de fan-fiction, las adaptaciones vernáculas, utilizan ambos elementos. De hecho, para una práctica creativa del TS es también necesario citar una gran variedad de evidencias textuales para justificar y explicar la expansión del original, asegurando la continuidad (Jenkins 2017).

Precisamente este foco en la creación de mundos con la práctica de la adaptación ha sido resaltado también en una de las más recientes publicaciones de la narratóloga Marie-Laure Ryan al respecto. La relación entre mundo y adaptación es, precisamente, la transmedialidad: "If transmedia is a new form of storytelling [...]. It should therefore hybridize the inherent transmediality of adaptation with the ihnerent world-expanding capacity of transmediality" (2017: 529).

Desde una perspectiva industrial, Clare Parody (2011) examina el rol de la adaptación de las franquicias de entretenimiento y la sitúa como un tipo diferenciado de adaptación, relacionándola asimismo con los universos y mundos ficcionales creados por la industria. Sitúa los proyectos de TS dentro de un concepto más amplio de franchise storytelling, compartiendo espacio con lo que llama un "palimpsesto de mundos narrativos" que se desarrollan a través del tiempo por medio de remakes y remediaciones de uno o varios textos ficcionales:

Franchise storytelling may be defined as the creation of narratives, characters, and settings that can be used both to generate and give identity to vast quantities of interlinked media products and merchandise, resulting in a prolonged, multi textual, multimedia fictional experience. (Parody 2011: 211)

Es decir, para la autora, las narrativas franquiciadas contienen tanto TS, como una creación coordinada de ficciones, como otras piezas que podríamos llamar cross media relativas a mundos narrativos, ofreciendo los ejemplos de algunas de las ficciones más populares de los siglos xx y xxI, James Bond, Star Wars, Harry Potter, El señor de los Anillos, Los piratas del Caribe, Batman o The Matrix.

La adaptación encuentra un gran desarrollo en estas franquicias, porque a) es muy útil en términos de rentabilidad económica e industrial, puesto que adapta textos conocidos y exitosos, con una base ya establecida de fans y b) porque ha probado que es un punto de arranque para franquicias transmediales alrededor de propiedades intelectuales populares como las mencionadas más 
arriba o los superhéroes originados en los cómics. De hecho, Parody relaciona estrechamente la adaptación con el TS:

\begin{abstract}
Adaptation and adaptations that are situated in a transmedia project are the products of a particular market strategy; they are contributing to and drawing on a particular kind of fictional experience and negotiating particular types of intertextual relation. (Parody 2011: 212)
\end{abstract}

Esta particularidad de la adaptación dentro del TS que la autora sitúa dentro de las franquicias de entretenimiento para ofrecer una perspectiva que tenga en cuenta los imperativos de la industria tiene varias facetas. Parody señala cómo el concepto de adaptación de Linda Hutcheon (2013: 16-21) privilegia un tipo de adaptación que contempla un diálogo bilateral con otro texto, en este caso único, frente al segundo modelo distinguido por la misma Hutcheon, el de la adaptación en diálogo con múltiples textos, que en el caso de las franquicias, y aquí es donde Parody difiere con Hutcheon, es la norma y no la excepción. Es decir, la adaptación dentro de las franquicias, y también dentro de los proyectos transmediales en ellas contenidos, dialogan con múltiples textos. Ello complica el análisis de su intertextualidad puesto que estos textos no tienen unas fronteras definidas, al referirse a una producción que siempre está en marcha y que deja un final abierto. Se refiere concretamente a la producción de las franquicias como "diaspórica" (2011: 212), no coordinada, con fronteras difusas en cuanto a la canonicidad de los textos y su autoría.

Siguiendo a Jenkins, Parody apunta que las franquicias adaptan mundos ficcionales antes que personajes o relatos, lo que explica también la predominancia de los géneros de ciencia ficción y fantasía en estas franquicias. Pero para Parody la diferencia fundamental en la adaptación dentro de las franquicias de entretenimiento es su relación con el desarrollo y dirección de la marca, ya que sus representaciones implican una propiedad intelectual y evocan en los consumidores esos mundos ficciones, todo ello relacionado con la creación de las llamadas experiencias totales de entretenimiento. Así:

\footnotetext{
"What franchise adaptations adapt" can therefore also be conceptualized as a brand identity, the intellectual property, advertising language, and presentational devices that cohere, authorize, and market the range of media products that together comprise the franchise experience. (Parody 2011: 214)
}

Estos mundos y personajes se convierten en parte de la imagen del estudio o conglomerado de medios a los que pertenecen y a su vez su imagen y sus aspectos reconocibles pasan a formar parte de los mismos. De hecho, según la autora, la adaptación puede contemplarse como una forma de gestionar la marca, ya que induce al recuerdo y a la nostalgia en los consumidores, y provoca un mayor reconocimiento de la misma.

Así pues, dentro de la cultura de la convergencia, la adaptación funciona dentro de las franquicias de entretenimiento normalizando la estética de la mul- 
tiplicidad (Parody 2011: 216), el reciclaje de contenido y textos que antes de ser imitativos son derivativos, no interpretativos o transformativos. Y estos textos, según la autora, no deben seguir siendo contemplados como productos de marketing, como paratextos antes que textos, sino que la práctica de la adaptación franquicial debe ser considerada asimismo una práctica creativa. Como puede percibirse, siendo muy interesante el subrayado del funcionamiento multilateral de la adaptación en la narrativa de las franquicias de entretenimiento, vuelve a quedar evidente la limitación del concepto de adaptación que se maneja en este caso, como "reciclaje" de contenidos "no interpretativos o transformativos".

\section{TEORÍA DE LAS NARRATIVAS TRANSMEDIALES: DEMEDIACIÓN, MUNDOS NARRATIVOS Y ADAPTACIÓN}

Recientemente recordábamos, siguiendo a Dominique Kalifa (2001) y a Kelleter (2017), que la industrialización de la cultura propia de fase postindustrial capitalista, con el cine como medio primordial en dicho sistema cultural, sigue en realidad basada en tres pilares fundamentales:
a) la novedad (para acceder al mercado un producto debe ser percibido como portador de algo nuevo); b) la serialización (para generar un buen retorno de una inversión, un producto de éxito debe ser repetido, con una ligera diferencia cada vez) y c) la adaptación (que es probablemente la manera más económica de combinar lo mejor de esas dos dimensiones, la novedad y la serialización). El cine no podía ser una excepción a esta regla y, de este modo, su lugar en esta red medial en continua expansión puede ser estudiada en dos direcciones. Por un lado, el cine es el resultado de múltiples adaptaciones. Por el otro, al mismo tiempo es el relevo o el punto de partida de innumerables otras expansiones y continuaciones, desde la fotografía de celebridades hasta los videojuegos, desde los cómics hasta las novelizaciones, desde las versiones de TV hasta las revistas de cotilleo, desde el póster al tráiler, desde los guiones publicados a las T-shirts, desde los parques de atracciones hasta los artículos académicos, etc. (Sánchez-Mesa y Baetens 2017b: 112)

Igualmente, desde hace unos años venimos planteando la necesidad de distinguir, al menos, dos dimensiones o modos distintos de entender y definir el funcionamiento de la transmedialidad: a) como cierta tendencia, inherente a la transmisión e historia de la comunicación cultural, de determinados contenidos, temas, mitos o personajes a aparecer en distintos medios o lenguajes; y b) el que dicha extensión se produzca de modo distribuido y organizado, en tanto "diseño de producción" de tal modo que este proceso coincida con la experiencia convergente que Jenkins identificó como TS.

Desde un punto de vista teórico, hemos subrayado también la importancia de marcar una diferencia entre ambas dimensiones de la transmedialidad y sus correspondientes procesos de transmedialización ya que, "si bien son, al mismo tiempo, bastante comparables, ya que ambos son síntomas de lo que Jenkins llamó cultura de la convergencia (2006), también son muy distintos, pues el modo 
en que descansan en la adaptación sigue orientaciones diferentes. Si en el primer caso nos encontramos con un empleo de la adaptación en un sentido bastante tradicional (una obra que existe en un determinado medio se convierte en otra cosa en un medio distinto), en el segundo, si bien no se carece por supuesto de una fuente, se arranca más bien de lo que podemos llamar un contenido demediado (Sánchez-Mesa y Baetens 2017a: 10-11). El término y concepto asociado lo tomamos de Garret Stewart (2011), quien lo aplicaba al cambio de funcionalidad del soporte libro en su transmedialización escultórica en bibliobjects. El concepto de demediación, o de contenido demediado, puede introducirse en la discusión sobre la teoría dominante del TS para señalar, teniendo en cuenta el énfasis de la noción de Stewart sobre la materialidad específica de cada medio (el libro como soporte material es transferido desde el medio literario al escultórico, como cuerpo o materia plástica), cierto efecto desmaterializador que dicho discurso del TS opera a partir de la idea de un contenido líquido o, en palabras del propio Jenkins, de "un flujo de contenidos a través de los medios" (Jenkins, 2009). Dicho de otro modo: "What the persistence of materiality in Stewart's definition stresses is the illusion that one can get rid of a medium's materiality" (Baetens y Sánchez-Mesa 2019).

Es evidente, a estas alturas, que el desplazamiento del foco desde el relato transmedial al mundo narrativo transmedial es una consecuencia de esta concepción abstracta del contenido, consolidado en definiciones influyentes como la de Klastrup y Pajares Tosca, para quienes entienden los transmedial storyworlds como "abstract content systems from which a repertoire of fictional stories can be actualized or derived across a variety of media forms" (2004: 409). Dicho desplazamiento, que para Espen Aarseth no sería sino la constatación de que esta dinámica industrial tiene que ver ante todo con la migración de las licencias o franquicias crossmedia (Aarseth 2006), habría sido ratificada por la principal teórica de la narratología transmedial, Marie-Laure Ryan, responsable de acuñar el sintagma "transmedial narrative worlds" (Ryan y Thön 2014). Como hemos señalado en alguna otra ocasión, diversos autores provenientes tanto de la narratología como de los Games Studies (Klastrup y Tosca 2004; Wolf 2011; Ryan 2014; Ryan y Thön 2014) han tratado de determinar tanto el concepto concreto de mundos transmediales, sus límites, los elementos que los componen y los procesos que se operan en ellos, por lo que se hace muy necesario elaborar una serie de herramientas concretas para analizarlos (Rosendo 2016: 66-67).

La consecuencia de todo este desplazamiento es, como hemos venido señalando, la preterición del componente narrativo de la idea dominante de transmedialidad, ya que la versión de nuevo cuño (radical) de la misma pasaría a depender de la construcción de esos storyworlds y no tanto del storytelling en sí mismo. El desplazamiento del discurso narrativo a los mundos transmediales como "contenedores" de múltiples relatos confirmaría, entre otras cosas, el predicamento cada vez más fuerte del paradigma de los videojuegos sobre la conceptualización del TS (Sánchez-Mesa et al. 2016), así como la centralidad de una de los fundamentos estéticos del lenguaje de los nuevos medios, según Lev 
Mánovich (2001), es decir, el imperio de la "lógica de la base datos" de la cual dependería la configuración de la mothership de un mundo transmedial.

En suma, si lo narrativo es ese "contenido abstracto que es constitutivo de la narratividad" o, en palabras de David Herman, son "blueprints for a specific mode of World-creation" (2009: 105), ¿cuál es la clave narrativa de las narrativas transmediales?, ¿puede ayudarnos la reflexión sobre el papel de la adaptación entre las estrategias de transmedialización a comprender mejor este (parcialmente) nuevo modo narrativo?

Sea como sea, debemos aclarar que, si bien la noción de transmedia storytelling cubre claramente los dos aspectos de la transmedialidad a los que no referíamos más arriba, es decir, (1) a la adaptación en sentido más tradicional por un lado, y (2) a la transmedialización del contenido demediado por el otro, no es menos cierto que las profundas implicaciones de esta distinción para la noción misma de relato (storytelling) no son siempre evidentes. En el primer caso, el TS estará más cercano a la adaptación de la historia, tal y como se da en las franquicias tradicionales. En el último caso, el TS tendrá más que ver con añadir una línea narrativa a un mundo narrativo. Y ello tendrá un impacto sobre la dimensión discursiva de la historia, sobre el foco o perspectiva narrativa, incluyendo la orquestación de voces y cosmovisiones colectivas, los lenguajes sociales o heteroglosia, que inevitablemente resultará menos elaborada con el fin de evitar todo lo posible las dificultades y roces intermediales propios de la migración a otros medios (Sánchez-Mesa y Baetens 2017a: 13).

2.1. La adaptación como herramienta de una estética de la resistencia intermedial

Precisamente uno de los aspectos que la adaptación puede iluminar en este debate, desde la perspectiva de los estudios de la intermedialidad es que si, como recuerda Jan Baetens, en la cultura de la convergencia: $1^{\circ}$ ) "todo puede migrar"; $2^{\circ}$ ) cualquier contenido "debe migrar", también se constata que, $3^{\circ}$ ) no todos los contenidos pueden "migrar completamente" (Baetens, 2016: 48). Es decir, el interés de esta mirada teórico-crítica, en un contexto que acepta la máxima de "If it doesn't spread, it's dead" (Jenkins et al. 2008) es la observación y el análisis atentos a las dificultades y rozamientos que esa migración transmedial debe afrontar en cada caso.

En efecto, la transmedialización en sentido radical, también experimenta fuerzas de resistencia, igual que las ha experimentado siempre la adaptación (transmedialización clásica). La especificidad mediática funciona así como una constricción, una suerte de obstáculo pero también un reto:

However, medium-specificity invites us to consider transmediality in terms of customisation, i.e. the specification of an idea, a work, a format, a genre etc., according to the properties of each medium. In other words, medium-specificity is a constraint; an obstacle as well as a challenger. (Baetens 2016: 48) 
En sintonía con Baetens, Rosendo (2019: 245-246) propone el sintagma constricción transmedial en la creación de contenidos para una narrativa transmedial desde una doble perspectiva: por un lado, narrativa, puesto que la narrativa específica creada para cada una de las plataformas no debe entrar en conflicto con las otras y debe estar en sintonía con la premisa que las unifica, y por otra mediática, que atiende tanto a necesidades de producción como expresivas.

Volviendo a Baetens, destaca que las dimensiones técnica y formal de la transmedialización son importantes pero igualmente lo son las histórica y cultural. De ahí que haya que tener en cuenta dos mecanismos más que vamos a relacionar con el papel de la adaptación, incluso diferencialmente:

[1] Transmedialisation always has a strong temporal dimension. It is less an event than a process [y $\left.2^{\circ}\right]$ Moreover, transmedialisation also has a strong 'networked' comparative dimension. It is less concerned with the shift from one medium to another (this is, perhaps, the specific angle of adaptation studies?) than the result of a wide and variegated range of relationships within a much larger network of related media, which all play a role in the process of transmedialisation. (Baetens 2016: 49)

Obviamente en los procesos de transmedialización hay "algo más" que adaptación, ya que la relación que se establece entre los distintos medios, como veíamos más arriba con Hutcheon y Parody, es más compleja y más multilateral que la que se da específicamente en la adaptación (incluso si esta no se reduce a menudo a la bipolaridad de dos textos), lo que daría pie a considerar, como hace Baetens, la oportunidad de invocar la noción de "serie cultural", en la versión de Dulac y Gaudreault (2004).

\section{AdAPTACIÓN, TRANSMEDIALIDAD Y NO FICCIÓN}

Otra de las llamadas de atención respecto a la reducción del modelo dominante de TS enunciadas desde el proyecto Nar-Trans es la formulación y mayoritaria aplicación de dicho modelo al dominio de la ficción, dejando en segundo plano precisamente todo un espacio creativo donde se están produciendo muchos de los desarrollos transmediales más interesantes, el de la llamada "no ficción" (Baetens, 2019). Esta proyección supone, entre otras cosas, una ampliación decidida de la teoría de la transmedialidad al periodismo y el documentalismo. En este dominio se produce "otro tipo de adaptación", a menudo más invisible o menos considerado, como es de la adaptación de los "hechos reales", acontecimientos o sucesos. De la consideración del papel del periodismo como hipotexto de adaptaciones cinematográficas basadas en hechos reales, ${ }^{4}$ pasaríamos, de nuevo de la mano de Jan Baetens, al estudio dos modos poco transitados y menos estudiados de periodismo en formato cómic y fotonovela. Y ello tratando

4 Para el caso concreto de la crónica de sucesos criminal en el cine español, véase Gómez y Sánchez-Mesa (2011). 
de dar respuesta a una pregunta que se nos antoja clave: "¿qué es un mundo narrativo en una narrativa transmedial de ficción y en una de no ficción?"5

El ejemplo del periodismo gráfico, según Baetens, supone un estímulo para colocar a la temporalidad en un lugar preeminente en el estudio de las narrativas transmediales. Al producirse la distribución de la historia en medios diferentes, dicha narrativa no es solo la consecuencia de una operación de marketing (ya se trate de una narrativa transmedial progresiva o del tipo "bola de nieve", ya sea diseñada ad hoc de manera distribuida), sino también "el resultado de diferencias más esenciales entre los medios, que simplemente no pueden combinarse de forma simultánea: el documental necesariamente sigue a la información periodística, aunque pueda relanzarla. Esta dimensión temporal merece ser estudiada en mayor profundidad en el campo de los estudios transmediales (2019: 263).

Junto a ese énfasis en la temporalidad o timeline de la narrativa transmedial, la diferencia fundamental entre el TS de ficción (canónico) y el de no ficción está en la noción de "mundo narrativo". A pesar de las dudas proverbiales y del debate teórico clásico sobre la imposibilidad de trazar fronteras nítidas entre ficción y documental, el modo de entender el llamado narrative story world sería distinto en uno y otro modo creativo y ello porque, como señala Baetens: "Además, en la ficción la narrativa transmedial se pone a menudo al servicio del mundo narrativo: lo que se cuenta no es tanto la historia en sí misma, sino una cierta forma de mostrar y explotar un mundo narrativo que con frecuencia está predefinido" (2019: 265).

En la no-ficción, por el contrario, la narrativa transmedial no cumple una función fundamental "complementaria", es decir, apoyar, expandir, distribuir, confirmar un mundo de ficción ya definido y preexistente, sino precisamente persigue distinguirse como "interpretación" de los hechos del mundo de no ficción sobre el que proyecto oponiéndose a lo que otros medios (distintos al periodismo) podrían hacer o hacen respecto a ese mundo de acontecimiento y hechos. Es una lógica de "oposición" que muestra lo que otros medios no hacen o no quieren hacer. Estas narrativas transmediales, así pues, configuran según Baetens un contradiscurso en relación a su mundo narrativo, es decir, respecto a la realidad. De ahí la importancia creciente, por ejemplo, del activismo transmedial en el desarrollo de un modo creativo tan pujante y con tantas posibilidades creativas y críticas como el documental interactivo o webdoc, con ejemplos clásicos entre las producciones internacionales como Highrise, ${ }^{6}$ The Quipu Project, ${ }_{1}^{7}$

\footnotetext{
${ }^{5}$ Baetens trabaja dos casos de periodismo gráfico: La grieta, un reportaje en forma de fotonovela de Guillermo Abril (texto) y Carlos Spottorno (fotografía) (La grieta, 2016) y Viva la vida, un cómic documental autobiográfico de Baudoin y Troubs (que elaboraron juntos tanto los dibujos como los textos) (Viva la vida. Los sueños de Ciudad Juárez, 2011).

${ }^{6}$ Metadocumental y proyecto de creación audiovisual (2010-2015) sobre la vida en los rascacielos de las grandes ciudades en el mundo, creado por Katerina Cizek y producido por el NFB, http:// outmywindow.nfb.ca/\#/outmywindow.
}

${ }^{7}$ Maria Court y Rosemarie Lerner (Estudio Chaka, 2015), https://interactive.quipu-project.com/\#/ en/quipu/intro. 
Refugee Republic ${ }^{8}$ o The Last Hijack, ${ }^{9}$ o, entre los españoles, proyectos como La primavera rosa, ${ }^{10}$ Las sinsombrero ${ }^{11}$ o Bugarach. Cómo sobrevivir al Apocalipsis. ${ }^{12}$

La constatación de la diferencia señalada aquí apoya la invitación a no reducir las narrativas transmediales a la ficción, porque el proceso de transmedialización funciona de modo diferente en este dominio de la no ficción y, por tanto, la "adaptación" también lo hace. La provocación de Baetens queda en el aire: ¿hay realmente un mundo narrativo en la narrativa de no ficción o nos enfrentamos a algo distinto cuando abordamos este tipo de narrativa? (Baetens 2019: 270).

\section{CONCLUSIONES}

Como hemos visto a lo largo de este artículo, en el (¿falso?) debate que prácticamente se inicia desde la primera definición de TS, los argumentos en contra de la adaptación como parte o práctica del mismo han partido de una concepción estrecha de la adaptación, considerando esta redundante o una herramienta para reutilizar contenidos. Christy Dena ha apuntado muy lúcidamente tres causas posibles para esta exclusión: la necesidad de diferenciar esta práctica de otras anteriores en un momento determinado de la industria; la apertura de un nuevo campo de estudio diferenciado de los establecidos estudios sobre la adaptación; y como herramienta pedagógica, para un primer contacto con lo que es un fenómeno que venimos estudiando desde hace menos de dos décadas.

Los argumentos a favor igualmente manejan un concepto de la adaptación que la separa de la llamada expansión o, como producto, llevándola a una función propiciadora, como punto de arranque de un proyecto transmedial. Tanto la diferenciación con la expansión como su función dentro de un proyecto, convierten la adaptación en una práctica diferenciada dentro de la transmedialidad, e incluso apuntan a una diferenciación entre distintos tipos de adaptación, lo que dificulta el estudio de narrativas transmediales complejas con múltiples puntos de entrada, o el estudio de narrativas transmediales complejas

\footnotetext{
${ }^{8}$ Martijn van Tol y Dirk Jan Visser (Submarine 2014) que muestra en una interfaz dinámica la vida en la ciudad en que se convierte el Camp Domiz (64.000 refugiados sirios), http://refugeerepublic. submarinechannel.com/.

9 Tommy Pallota y Felke Volking (Submarine Channel 2014) narra la actividad y presenta la posibilidad de explorar en el contexto de secuestros de barcos por los piratas somalíes, https:// lasthijack.com/.

10 Serie de documentales interactivos sobre la situación de la comunidad LGTBI en el mundo (2015-2019) de Mario de la Torre (Creta Prod., Pasajes invisibles y Malvalanda), http://www.laprimaverarosa.com/.

11 Una de las producciones de la factoría del RTVE Lab (Laboratorio de innovación audiovisual), creada por Serrana Torres, Tània Balló y Manuel Jiménez, que plantea un proyecto educativo transmedial para la recuperación de las figuras femeninas de la Generación del 27, http://www.rtve.es/ lassinsombrero/es/webdoc/.

12 Un docugame sobre el bizarro caso de lo sucedido en el pequeño pueblo francés de Bugarach ante la inminencia de un supuesto apocalipsis. Producido por Arnau Gifreu (RTVE Lab, Nanouk Films, Interactius y Playfilm), http://lab.rtve.es/webdocs/bugarach/.
} 
que formen parte de franquicias de entretenimiento. Aunque el enfoque aquí expresado se centra en la producción y el diseño, precisamente es necesario un replanteamiento de la adaptación dentro de la transmedialidad para poder comprender mejor cómo tanto estas ficciones como los relatos factuales transmediales son recibidos por la audiencia, y cómo esta, dentro de la cultura de la convergencia, contribuye a ellos. Por otra parte, es muy destacable que todos los autores considerados señalen, en cierta medida, la necesidad de estudiar la transmedialidad en contacto con los estudios sobre adaptación, eliminando una barrera que obedece más a una delimitación de disciplinas que a su objeto de estudio.

Y, por último, si en esta revisión ha quedado claro que el concepto y la práctica de la adaptación no solo no es ajena, sino que es primordial para las prácticas y procesos de transmedialización, no lo es menos que las novedades que comporta este modo de diseño narrativo tienen también sus consecuencias sobre la adaptación como fenómeno complejo de transferencia de contenidos entre medios. Hemos visto cómo la diferencia suele establecerse en el par "extensión/adaptación" pero la pregunta es, ¿puede hacerse una extensión (precuela, secuela, desarrollo de un intersticio no desarrollado por el texto adaptado, etc.) sin una suerte de adaptación del material de partida? $\mathrm{O}$, dicho de otro modo, en un diseño de producción radicalmente distribuido, si hay un timeline un "viaje del usuario" (user's journey) y una apuesta por el medio concreto desde el que se lanza ese storyworld, al ser un fenómeno temporal, un proceso y no solo un producto, ¿nos encontramos ante una práctica, quizás distinta, con unas funciones particulares (¿nuevas?) de la adaptación? Esperamos que este artículo contribuya a poner las bases para acometer la respuesta a dicha pregunta a partir de nuestra revisión conceptual y crítica de un debate (transmedia storytelling vs. adaptación) que consideramos tan sintomático del campo teórico sobre las narrativas transmediales como agotado.

\section{OBRAS CITADAS}

Aarseth, Espen (2006): "The Culture and Business of Crossmedia Production", Popular Communication, vol. 4, n. ${ }^{\circ}$ 3, pp. 203-211.

Baetens, Jan (2016): "Between Adaptation, Intermediality and Cultural Series: the Example of the Photonovel". En D. Sánchez-Mesa, J. Alberich-Pascual y N. Rosendo (coords.): "Transmedia narratives", Artnodes n. ${ }^{\circ}$ 18, pp. 47-55. Doi: http://dx.doi.org/10.7238/a. v0i18.3053.

— (2019): "Mundos narrativos de ficción y de no ficción. Apuntes sobre las narrativas transmediales en los cómics y fotonovelas periodísticos". En D. Sánchez-Mesa (ed.): Narrativas transmediales. Las metamorfosis del relato en los nuevos medios digitales. Barcelona, Gedisa, pp. 259-271.

Baetens, Jan; Sánchez-Mesa, Domingo (2015): "Literature in the Expanded Field: Intermediality at the Crossroads of Literary Theory and Comparative Literature", Interfaces. 
Image Texte Language vol. 36. Accesible en <https://preo.u-bourgogne.fr/interfaces/index.php?id=245> [última visita: 8.2.2019].

_ (2019): "A Note on Demediation: From Book Art to Transmedia Storytelling", Leonardo, vol. $52, n .^{\circ} 3$.

Bourdaa, Mélanie (2013): "Following the Pattern: The Creation of an Encyclopaedic Universe with Transmedia Storytelling", Adaptation, vol. 6, n. ${ }^{\circ}$ 2, pp. 201-214.

Dena, Christy (2009): Transmedia Practice; Theorising the Practice of Expressing a Fictional World across Distinct Media and Environments. Tesis doctoral. University of Sydney. Accesible en: <http://www.christydena.com/phd/> [última visita: 8.2.2019].

— (2018): "Transmedia Adaptation: Revisiting the No-Adaptation Rule". En: The Routledge Companion to Transmedia Studies. Londres: Routledge, pp. 195-206.

Dulac, Nicolas; Gaudreault, André (2004): "Heads or Tails: The Emergence of a New Cultural Series, from the Phenakisticope to the Cinematograph", Invisible Culture. An Electronic Journal for Visual Culture, n. ${ }^{\circ}$ 8. Accesible en <http://www.rochester.edu/ in_visible_culture/Issue_8/issue8_dulac-gaudreault.pdf> [última visita: 8.2.2019].

Evans, Elizabeth (2011): Transmedia television: Audiences, new media, and daily life. Londres, Routledge.

Genette, Gérard (1982): Palimpsestos. La literatura en segundo grado. Madrid, Taurus.

Gomez, Jeff (2007): "8 Defining Characteristics of Transmedia Production". En: Creating Blockbuster Worlds event, recogido por Christy Dena. En línea: <http://www.christydena.com/2007/10/jeff-gomezs-8-defining-characteristics-of-transmedia-production> [última visita: 8.2.2019].

Herman, David (2009): Basic Elements of Narrative. New Jersey, Wiley Editorial.

- (2013): "Cognitive Narratology", The Living Handbook of Narratology. En línea: <http:// wikis.sub.uni-hamburg.de/lhn/index.php/Cognitive_ Narratology> [última visita: 6.2.2019].

Hutcheon, Linda; O'Flynn, Siobhan (2013): A Theory of Adaptation. $2^{\text {a }}$ ed., Nueva York, Routledge.

Jenkins, Henry (2003): "Transmedia Storytelling. Moving Characters from Books to Films to Video Games Can Make them Stronger and more Compelling, MIT Technology Review. En línea: <http://www.technologyreview.com/news/401760/transmediastorytelling> [última visita: 6.2.2019].

(2008): Convergence Culture. La cultura de la convergencia en los medios de comunicación [2006]. Barcelona, Paidós.

(2009): "The Revenge of the Origami Unicorn: Seven Principles of Transmedia StoryteIling", Confessions of an Aca-Fan (The Official Weblog of Henry Jenkins). En línea: <http://henryjenkins.org/2009/12/the_revenge_of_the_origami_uni.html> [última visita: 16.2.2019].

(2011): "Transmedia 202: Further Reflections". Confessions of an Aca-Fan (The Official Weblog of Henry Jenkins). En línea: <http://henryjenkins.org/blog/2011/08/defining_transmedia_further_re.html> [última visita: 16.2.2019].

_ (2017): "Adaptation, Extension, Transmedia", Literature/Film Quaterly, vol. 45, n. ${ }^{\circ} 2$. En línea: <https://lfq.salisbury.edu/_issues/first/adaptation_extension_transmedia. html> [última visita: 16.2.2019]. 
Jenkins, Henry; Li, Xiaochang; Krauskopf, Ana Domb; Green, Joshua (2008): If it doesn't spread, it's dead: Creating value in a spreadable marketplace. Massachusetts, Convergence Culture Consortium, CMS, MIT. Accesible en <http://convergenceculture. org/research/Spreadability_doublesidedprint_final_063009.pdf> [última visita: 2.2.2019].

Kalifa, Dominique (2001): La culture de masse en France 1: 1860-1930. París, La Découverte. Kelleter, Frank (ed.) (2017): Media of Serial Narrative. Columbus, Ohio State University Press. Kinder, Marsha (2014): "Medium Specificity and Productive Precursors". En M. Kinder y T. McPherson (eds.): Transmedia Frictions. The Digital, the Arts and the Humanities. Oakland, University of California Press.

Klastrup, Lisbeth; Pajares Tosca, Susana (2004): "Transmedial Worlds: Rethinking Cyberworld Design", Proceedings of the International Conference on Cyberworlds IEEEE Computer Society. En línea: <http://www.itu.dk/people/klastrup/klastruptosca transworlds.pdf> [última visita: 8.12.2016].

Long, Geoffrey A. (2007): Transmedia Storytelling: Business, Aesthetics and Production at the Jim Henson Company. Tesis doctoral, Massachusetts Institute of Technology. Accesible en: <https://cmsw.mit.edu/transmedia-storytelling-jim-henson-company/> [última visita 8.2.2019].

Manovich, Lev (2001): The Languages of New Media. Cambridge, Mass., MIT Press.

Parody, Clare (2011): "Franchising/Adaptation". En Adaptation Vol 4 no 2, pp 210-218.

Pratten, Robert (2015): Getting Started in Transmedia Storytelling. A practical Guide for Beginners [2011]. $2^{\mathrm{a}}$ ed. Londres, Amazon.

Producers Guild of America (2010): "Code of Credits: New Media" (Transmedia producer). En línea: <https://www.producersguild.org/page/coc_nm\#transmedia> [última visita 8.2.2019].

Rosendo Sánchez, Nieves (2016): "Mundos transmediales: revisión conceptual y perspectivas teóricas del arte de crear mundos", Revista ICONO14. Revista científica de Comunicación y Tecnologías emergentes, vol. 14, n. 1, pp. 49-70. Doi: https://doi. org/10.7195/ri14.v14i1.930.

- (2019): "Dinámicas de la creación de un universo transmedia. La expansión de El proceso, de Kafka". En D. Sánchez-Mesa (ed.): Narrativas transmediales. Las metamorfosis del relato en los nuevos medios digitales. Barcelona, Gedisa, pp. 233-249

Ryan, Marie-Laure (2017): "Transmedia Storytelling as Narrative Practice". En T. Leitch (ed.): The Oxford Handbook of Adaptation Studies. Oxford, Oxford University Press.

Ryan, Marie-Laure; Thon, Jan-Nöel (eds.) (2014): Storyworlds accross media. Toward a Media-Conscious Narratology. Lincoln/Londres, Nebraska Univ. Press.

Sánchez-Mesa, Domingo (ed.) (2019): Narrativas transmediales. Las metamorfosis del relato en los nuevos medios digitales. Barcelona, Gedisa.

Sánchez-Mesa, Domingo; Aarseth, Espen; Pratten, Robert; Scolari, Carlos A. (2016): "Transmedia (Storytelling?): A polyphonic critical review". En D. Sánchez-Mesa, J. Alberich-Pascual y N. Rosendo (coords.): "Transmedia narratives", Artnodes, n. 18, pp. 8-19. Doi: http://dx.doi.org/10.7238/a.v0i18.3064.

Sánchez-Mesa, Domingo; Baetens, Jan (2017a): "La literatura en expansión, intermedialidad y transmedialidad en el cruce entre la literatura comparada, los estudios culturales 
y los New Media Studies", Tropelías. Revista de Teoría de La Literatura y Literatura Comparada, n. ${ }^{\circ} 27$, pp. 6-27.

- (2017b): "La Ventana indiscreta de Alfred Hitchcock como cineromanzo. La adaptación como tansmedialización", Tropelías. Revista de Teoría de La Literatura y Literatura Comparada, n. ${ }^{\circ} 28$, pp. 110-124. Doi: https://doi.org/10.26754/ojs_tropelias/tropelias.2017282060.

Scolari, Carlos A. (2009): "Transmedia Storytelling: Implicit Consumers, Narrative Worlds and Branding in Contemporary Media Production", International Journal of Communication, n. ${ }^{\circ}$ 3, pp. 586-606.

- (2013): Narrativas transmediales. Cuando todos los medios cuentan. Bilbao, Deusto.

- (2012): "The Triplets and the Incredible Shrinking Narrative. Playing in the Borderland between Transmedia Adaptation". En I. Ibrus y C. A. Scolari (eds.): Crossmedia Innovations. Texts, Markets, Institutions. Frankfurt, Peter Lang, pp. 45-60.

Stewart, Garrett (2011): Bookwork. Medium to Object to Concept to Art. Chicago, Chicago University Press. 